\title{
Temporal Adaptive Changes in Contractility and Fatigability of Diaphragm Muscles from Streptozotocin-Diabetic Rats
}

\author{
Marco Brotto, ${ }^{1}$ Leticia Brotto, ${ }^{1}$ J.-P. Jin,, ${ }^{2}$ Thomas M. Nosek, ${ }^{3}$ and Andrea Romani ${ }^{3}$ \\ ${ }^{1}$ Muscle Biology Research Group (MUBIG), Schools of Nursing \& Medicine, University of Missouri-Kansas City, \\ Kansas City, MO 64108, USA \\ ${ }^{2}$ Department of Physiology, School of Medicine, Wayne State University, Detroit, MI 48201, USA \\ ${ }^{3}$ Department of Physiology \& Biophysics, School of Medicine, Case Western Reserve University, 10900 Euclid Avenue, \\ Cleveland, $\mathrm{OH}$ 44106, USA
}

Correspondence should be addressed to Andrea Romani, amr5@po.cwru.edu

Received 21 December 2009; Accepted 18 February 2010

Academic Editor: Guy M. Benian

Copyright () 2010 Marco Brotto et al. This is an open access article distributed under the Creative Commons Attribution License, which permits unrestricted use, distribution, and reproduction in any medium, provided the original work is properly cited.

\begin{abstract}
Diabetes is characterized by ventilatory depression due to decreased diaphragm (DPH) function. This study investigated the changes in contractile properties of rat DPH muscles over a time interval encompassing from 4 days to 14 weeks after the onset of streptozotocin-induced diabetes, with and without insulin treatment for 2 weeks. Maximum tetanic force in intact DPH muscle strips and recovery from fatiguing stimulation were measured. An early (4-day) depression in contractile function in diabetic DPH was followed by gradual improvement in muscle function and fatigue recovery ( 8 weeks). DPH contractile function deteriorated again at 14 weeks, a process that was completely reversed by insulin treatment. Maximal contractile force and calcium sensitivity assessed in Triton-skinned DPH fibers showed a similar bimodal pattern and the same beneficial effect of insulin treatment. While an extensive analysis of the isoforms of the contractile and regulatory proteins was not conducted, Western blot analysis of tropomyosin suggests that the changes in diabetic DPH response depended, at least in part, on a switch in fiber type.
\end{abstract}

\section{Introduction}

Increased fatigability of skeletal muscles, characterized by difficulty in squatting and in performing repetitive motions, is commonly observed in both type I and type II diabetes. Insulin-dependent diabetes mellitus (IDDM) can also lead to ventilatory depression and decreased sensitivity to hypercapnia [1]. Diaphragm function is a limiting factor for overall muscle performance, in as much as intense physical activity causes fatigue of diaphragm muscle even in healthy individuals of varying fitness levels [2]. As for the cause of the observed weakness in skeletal and respiratory muscles in diabetics, it has been generally attributed to the motor and sensory neuropathy [3] often present in diabetic patients. Yet, modifications in nerve function and conductance are not consistently observed in these patients, and when they do appear, they usually do so at a later stage of the disease, thus implying that diabetic neuropathy cannot be the only cause of muscular dysfunction [4].
An increased susceptibility to muscle fatigue not only affects the quality of life but also reinforces the tendency to physical inactivity typical of the diabetic patient. Decreased physical activity is very deleterious since even moderate exercise is beneficial in controlling glycemia in diabetic patients, limiting the dependence on insulin or oral antidiabetic drugs [5]. In recent years, the lack of or a decrease in physical activity has been considered one of the main causes, if not a direct trigger, of the increased incidence in chronic diseases such as diabetes, obesity, hypertension, and other cardiovascular disorders [6]. Therefore, understanding factors that in addition to inactivity limit exercise tolerance in diabetic patients is very important.

Whilst several studies have focused on understanding how muscle contractility exerts its regulatory role on glycemia under physiological and diabetic conditions [1-3], little is known about the cellular mechanisms responsible for the increased muscle fatigability under diabetic conditions. Moreover, the majority of the studies performed to date 
have been carried out in hind-limb skeletal muscles or in cardiac tissue [7-11] whereas scarce attention has been paid to diaphragm muscle performance. In two previous studies in diaphragm muscles, Hida et al. [12] reported a worsening of diaphragm contractile function at 3 and 7 days after streptozotocin administration, while McGuire and MacDermott [13] reported a compromised contractile function in diabetic diaphragm muscles at $\sim 3$ months. To our knowledge, neither of these two studies, nor any other study to date, has investigated the effects of diabetes on diaphragm muscles within a sequential time frame that encompasses acute, steady-state, and chronic effects of diabetes.

Hence, the main aim of our study was to investigate changes induced by diabetes in diaphragm contractility and fatigability in a system in which alterations in nerve function and conductivity have no direct impact on muscle performance throughout a broad time span. To achieve our aim, we used streptozotocin- (STZ-) induced diabetic rats and age-matched controls over a period ranging from 4 days to 14 weeks after STZ administration. The effect of insulin supplementation at a later time point ( 12 weeks STZ-diabetic +2 weeks of insulin treatment) was also investigated. We utilized intact diaphragm muscle to concomitantly study maximal tetanic force produced by the muscles as well as the contractile response to, and recovery from, fatiguing stimulation. Skinned fibers were used to assess changes in calcium sensitivity.

Our data demonstrate that there is an early acute, deleterious effect of diabetes on muscle function. This phase is followed first by a gradual improvement in muscle function reflecting a compensatory adaptation that peaks at $\sim 8$ weeks and then a marked deterioration in muscle function at 14 weeks after diabetes onset. Two weeks of insulin treatment at 12 weeks after STZ administration completely reversed the compromised muscle function observed at 14 weeks. A similar bimodal pattern of response was also observed in contractility studies conducted in skinned diaphragm muscle fibers.

These results are discussed in a context of clinical relevance as the time frame observed (days to weeks in rats) would be approximately equivalent to changes that would occur over months to several years of pathology in humans. Our results suggest that changes in diaphragm contractile machinery composition and function play a role in the pathophysiology of respiratory diseases secondary to diabetes in addition to limiting physical activity in diabetic patients.

\section{Materials and Methods}

2.1. Animals and Euthanasia. Male Sprague Dawley rats weighting 180-210 g (Harlan Laboratories, Indianapolis, IN) were sacrificed by $\mathrm{CO}_{2}$ inhalation. All procedures were performed in accordance with the "Guiding Principles in the Care and Use of Animals" approved by the American Physiological Society and the Animal Protocol Review Committee of the School of Medicine at CWRU.
2.2. Materials. Insulin and glucose-reader strips were from Gemco (Hudson, OH). Urine glucose/ketones strips were from Fisher (Pittsburgh, PA). Streptozotocin and all other chemicals were of analytical grade (Sigma, St Louis, MO).

2.3. Induction of Diabetes. Rats were randomly divided into two groups. One group of animals was rendered diabetic by a single intraperitoneal (ip) injection of $65 \mathrm{mg} / \mathrm{kg}$ streptozotocin (STZ) in citrate buffer ( $\mathrm{pH} 4.0$ ), while control animals received an equivalent volume of vehicle. Diabetes onset occurred within 48 hours following STZ administration and it was determined by the appearance of glucose in the urine with glucose strips. The diabetic animals and the agematched controls were sacrificed at 3-4 days (referred to as 4day animals for simplicity), 2 weeks, 4 weeks, 8 weeks, and 14 weeks after STZ administration. After 12 weeks from diabetes onset, a subgroup of diabetic animals were placed on daily ip injection of Ultralente insulin ( $2 \mathrm{U} / 50 \mathrm{~g}$ body weight). These animals were sacrificed at 14 weeks from diabetes induction (i.e., after 2 weeks of insulin treatment).

2.4. Intact Muscle Preparation. These experiments were performed following the protocols established by de Paula Brotto et al. [14]. After the animals were sacrificed, the intact diaphragm (DPH) muscles were removed and placed in a dissecting dish containing a modified Ringer solution having the following composition $(\mathrm{mM}): 136.5 \mathrm{NaCl}, 5.0$ $\mathrm{KCl}, 1.8 \mathrm{CaCl}_{2}, 0.4 \mathrm{NaH}_{2} \mathrm{PO}_{4}, 0.5 \mathrm{MgCl}_{2}, 11.9 \mathrm{NaHCO}_{3}$, and 10 glucose, $\mathrm{pH} 7.4$, continuously bubbled with $95 \% \mathrm{O}_{2}$, $5 \% \mathrm{CO}_{2}, 25^{\circ} \mathrm{C}$, to establish control, normoxic conditions. DPH muscles were carefully dissected into small muscle strips measuring $35-40 \mathrm{~mm}$ in length and less that $1 \mathrm{~mm}$ in thickness to favor optimal oxygen diffusion throughout the muscle strips [14]. For each experimental data point 23 strips per animals and 3-6 animals per group (diabetic or nondiabetic) were utilized, and the obtained data were averaged. DPH muscle strips were mounted vertically from the central tendon to the rib cage tissue on a Radnoti (Monrovea, CA) system. The muscle sutures were attached to an isometric force transducer and to a stationary post. The output of the force transducer was digitized and stored in a computer for later analysis. The resting tension and the stimulatory voltage (provided by a Grass digital stimulator) were adjusted to produce maximal isometric tetanic force $\left(T_{\max }\right)$. The stimulation pattern for these experiments was completely automated after the muscles were mounted. Powerlab/400 E series (ADInstruments, Mountain View, CA) was used to drive a digital Grass stimulator and Chart for Windows v4.0.1 (ADInstruments, Mountain View, CA) was used to collect, digitize, analyze, and store the data to a PC.

2.5. Intermittent Fatiguing Protocols. After $T_{\max }$ was determined, the intact DPH strips were allowed to equilibrate for 20 minutes in the Ringer's solution described above (Equilibration Period, Equil in Figure 1). During equilibration, muscle strips were stimulated with $100 \mathrm{~Hz}, 330 \mathrm{~mA}, 500 \mathrm{msec}$ electrical pulse-train administered with a periodicity of 1 minute to generate $T_{\max }$. Stimulation during equilibration 


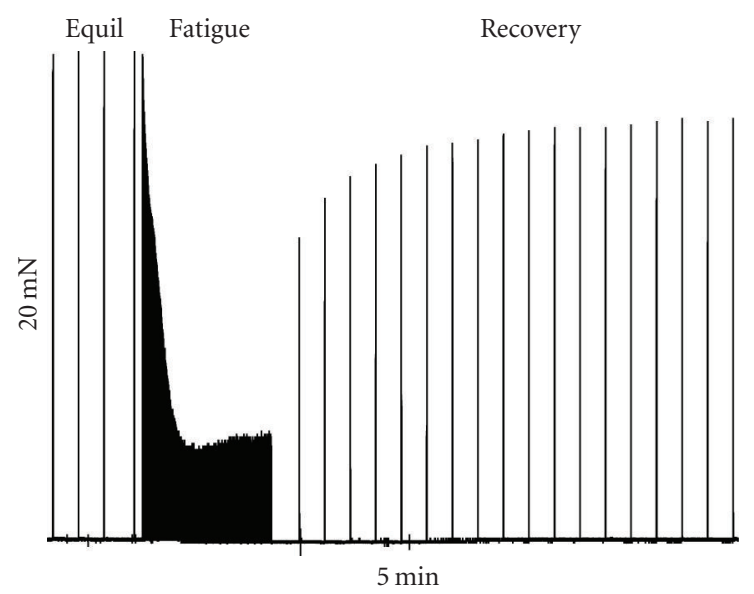

FIGURE 1: Original raw digital tracing of a control DPH muscle strip showing our intact contractility protocol. This intact contractility record shows the last 4 minutes of equilibration (Equil), followed by the fatiguing stimulation period (Fatigue, 5 minutes) and by the recovery period. During Equil and Recovery, the muscle strip was stimulated with tetanic contractions every minute, while during the fatigue period, the muscles were stimulated every second. Calibration bars for this specific experiment are shown. The horizontal bar denotes time in minutes and the vertical bar the relative force in $\mathrm{mN}$ produced by the preparation.

allowed us to monitor the condition of the muscle and did not cause measurable fatigue over the 20 -minute equilibration period. Following equilibration, the muscles from the fatigue group were subjected to a 5-minute fatiguing protocol (Fatiguing Period, Fatigue, Figure 1) consisting of the same stimulatory pattern administered at a 1-second periodicity. Thereafter, the periodicity of the stimulus train was returned to 1-minute intervals and the muscles were allowed to recover for 30 minutes (Recovery Period, Figure 1). All force data were normalized to the last tetanic contraction at the end of the equilibration period and just prior to the start of the fatiguing protocol (this $T_{\max }=100 \%$ ). Absolute force, normalized per cross-sectional area (in $\mathrm{N} / \mathrm{cm}^{2}$ ), was determined at the end of the equilibration period as previously described [14].

2.6. Triton-Skinned Muscle Fibers. Force-versus-pCa relationships were measured using skinned muscle preparations dissected from the same DPH strips used for the intact muscle experiments described above. The technique has been described in detail elsewhere $[15,16]$. In brief, single muscle fibers were dissected under a microscope and mounted between an isometric force transducer and a stationary post. All membranes were removed by exposure to Triton X100. Fibers were activated by transferring them to troughs containing different amounts of free calcium. Calciumactivated force was recorded and normalized to the crosssectional area of each fiber (in $\mathrm{N} / \mathrm{cm}^{2}$ ) and to the maximal force produced by each fiber $\left(100 \%, F_{\max }\right)$. The force-versuspCa curve for each fiber was fit to Boltzmann-Sigmoidal equation as previously described $[15,16] . \mathrm{Ca}^{2+}$ sensitivity was evaluated from $\mathrm{Ca}_{50}$ (the $\mathrm{Ca}^{2+}$ concentration producing halfmaximal force). The steepness of the curve was evaluated from $N$, the Hill coefficient. After each parameter was obtained for individual fibers, an average force versus $\mathrm{pCa}$ was calculated. In Table $1, \mathrm{n}$ represents the number of individual muscle preparations studied while the number in parenthesis indicates the number of animals from which the fibers were isolated.

2.7. Western Blot Analysis of Tropomyosin Isoforms. The rat skeletal muscle SDS-gel samples were resolved by SDS-PAGE using 14\% Laemmli gels with an acrylamide: bisacrylamide ratio of $180: 1$ cast using a Bio-Rad mini-Protean II system. The resolved protein bands were electrically transferred to nitrocellulose membrane $(0.45 \mu \mathrm{m}$ pore size $)$ using a BioRad semidry transfer apparatus at $5 \mathrm{~mA} / \mathrm{cm}^{2}$ for 15 minutes. The membrane was blocked in Tris-buffered saline (TBS) composed of (in mm) $137 \mathrm{NaCl}, 5 \mathrm{KCl}$, and 25 Tris- $\mathrm{HCl}$ ( $\mathrm{pH}$ 7.4) containing $1 \%$ BSA at room temperature for 1 hour and incubated with monoclonal antibody $(\mathrm{mAb}) \mathrm{CH} 1$ against $\alpha$ - and $\beta$-Tm mixed with mAb CG3 against $\gamma$-Tm (provided by Dr. Jim Lin, University of Iowa) [17] in TBS containing $0.1 \% \mathrm{BSA}$ at $4{ }^{\circ} \mathrm{C}$ overnight. After three washes with TBS containing $0.5 \%$ Triton X-100 and $0.05 \%$ SDS and two TBS rinses, the membrane was incubated with alkaline phosphatase-conjugated antimouse IgG second antibody (Sigma) in TBS containing $0.1 \%$ BSA at room temperature for 1.5 hours. After washes as above, 5-bromo-4-chloro-3indolyl phosphate/nitro blue tetrazolium substrate reaction was carried out as described previously [17] to visualize the tropomyosin isoforms.

2.8. Statistical Analyses. Values are mean \pm SD. Significance was determined by ANOVA followed by either Tukey's or Bonferroni's tests. A value of $P<.05$ was used as criterion for statistical significance.

\section{Results}

At the time of sacrifice, the level of glycemia was measured by glucose-strip reader on $25 \mu \mathrm{l}$ of blood and found to be $425 \pm 15$ versus $88 \pm 5 \mathrm{mg} / 100 \mathrm{~mL}$ for 2 -week diabetic and age-matched control animals, respectively $(n=12$ for both experimental groups, $P<.05$ ), not increasing significantly with diabetes progression (e.g., $459 \pm 17$ versus $93+$ $7 \mathrm{mg} / 100 \mathrm{~mL}$ in 4 -week diabetic and age-matched control animals, respectively ( $n=10$ for both experimental groups, $P<.05)$. Rats receiving insulin supplementation presented a glycemia of $98 \pm 10 \mathrm{mg} / 100 \mathrm{~mL}$ at the time of sacrifice as compared to $96 \pm 6$ and $461 \pm 11 \mathrm{mg} / 100 \mathrm{~mL}$ in age-matched control and 14-week diabetic animals, respectively $(n=6$ for all experimental groups).

Maximal tetanic force ( $T_{\max }$, normalized to crosssectional area in $\mathrm{N} / \mathrm{cm}^{2}$ at the end of the equilibration period) was significantly reduced by $\sim 25 \%$ for muscles isolated from 4-day diabetic animals compared with agematched controls (see Table 1). Figure 2(a) illustrates the response of the 4-day diabetic and age-matched control 


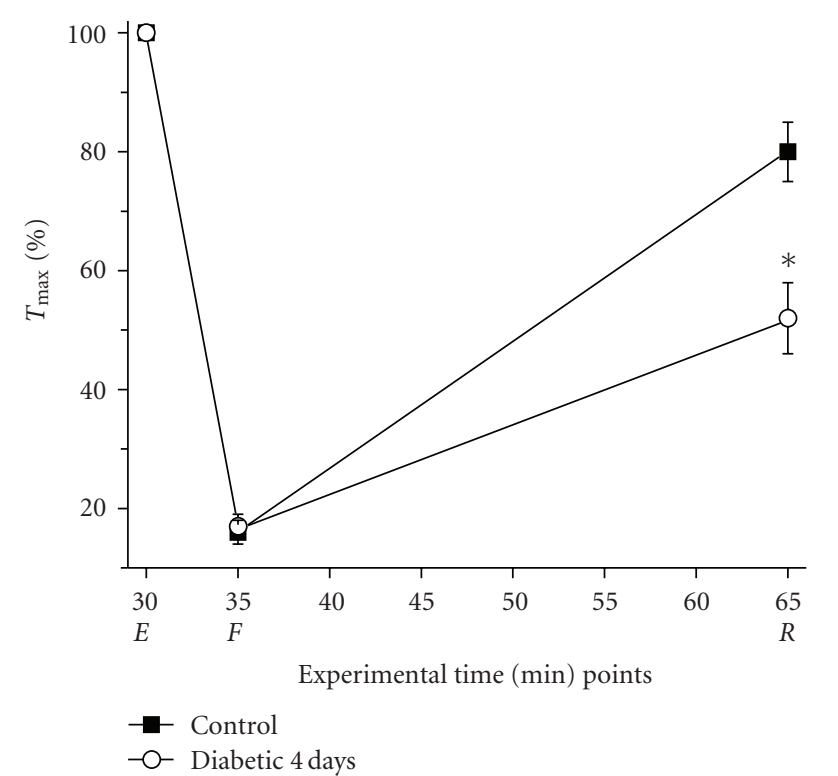

(a)

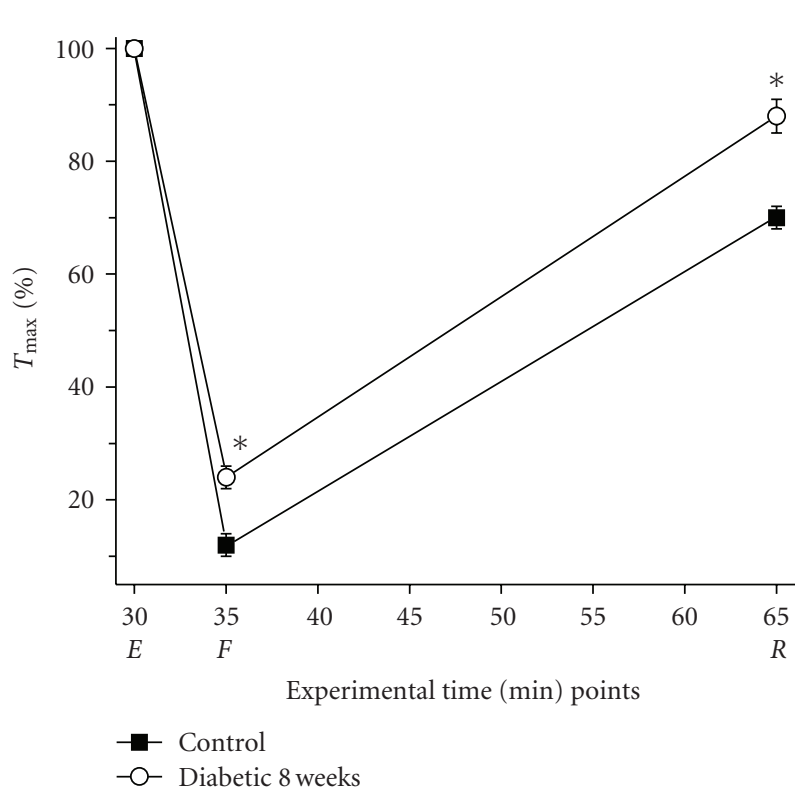

(c)

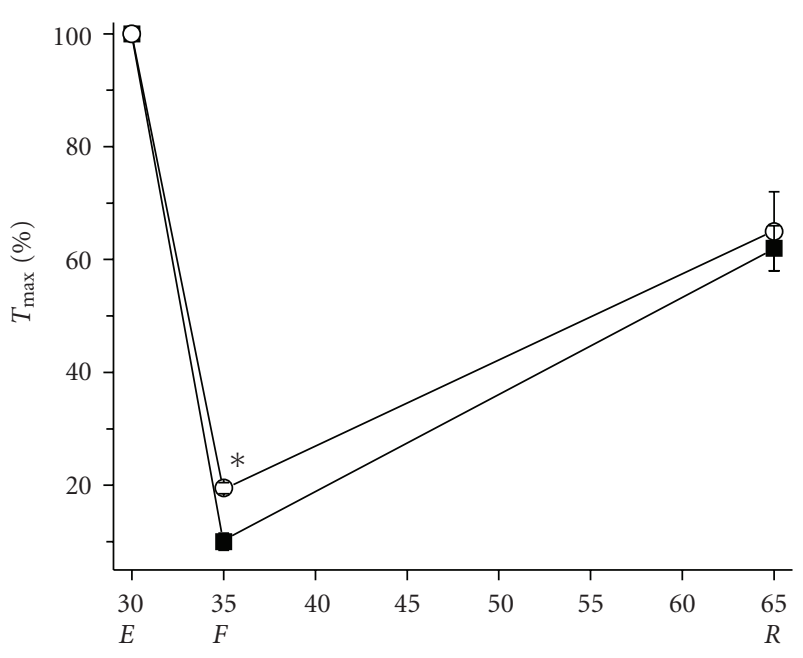

Experimental time (min) points

- Control

$-O-$ Diabetic 4 weeks

(b)

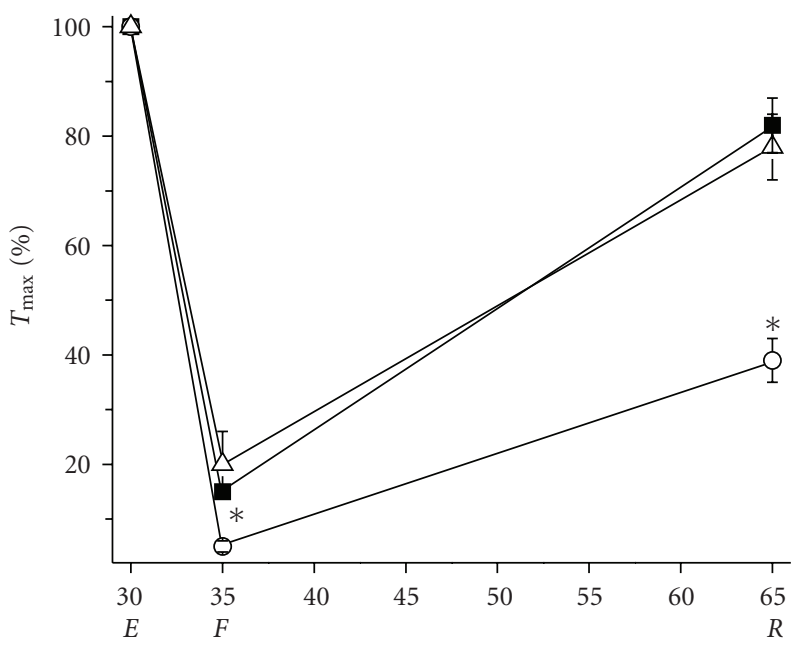

Experimental time (min) points

$$
\begin{aligned}
& - \text { - Control } \\
& -\bigcirc-\text { Diabetic } 14 \text { weeks } \\
& \triangle-\text { Insulin-treated }
\end{aligned}
$$

(d)

FIgURE 2: Temporal adaptation of intact diaphragm (DPH) muscle contractile function in diabetic muscles. All data are presented as the mean \pm SD. (a) Diabetes exerts an acute deleterious effect on DPH contractile function that is reverted with time (b and c) and profoundly deteriorates with the progression of diabetes (d). After 2 weeks of insulin treatment DPH contractile function significantly improves to levels comparable to control levels (d). In all panels, closed squares show data for control muscles, open circles for diabetic muscles, and the open triangle represents the diabetic + insulin treatment. ${ }^{*}$ means $\mathrm{P}<.05 . T_{\max }$, number of experiments and statistical details are presented in Table 1 and methods.

muscles to the fatiguing protocol, with all data normalized to $T_{\max }$ for each muscle. While there was an identical decrease in force in both muscle sets at the end of the fatigue period to approximately $15 \%$ of $T_{\max }$, the diabetic muscles regained force to only $52 \%$ of $T_{\max }$ after 30 minutes of recovery compared to $80 \%$ for the age-matched controls. The acute effect of diabetes on the recovery of intact muscle strip function to fatiguing stimulation at 4 days post streptozotocin administration suggests that acute loss of insulin signaling renders diaphragm muscle susceptible to fatigue-induced damage.

We found that the diaphragm muscle of 4-week diabetic animals may have adapted to the high glucose levels (Table 1, Figure 2(b)). $T_{\max }$ was significantly increased from the value in 4-day diabetic animals and was only slightly reduced (by approximately 10\%) from the value in age-matched 
TABLE 1: Contractile data from diaphragm of diabetic animals and age-matched controls.

\begin{tabular}{|c|c|c|c|c|c|c|c|c|c|}
\hline \multicolumn{10}{|c|}{ Intact muscles } \\
\hline & \multicolumn{2}{|c|}{4 days } & \multicolumn{2}{|c|}{4 weeks } & \multicolumn{2}{|c|}{8 weeks } & \multicolumn{3}{|c|}{14 weeks } \\
\hline & $\begin{array}{l}\text { Control } \\
n=7(4)\end{array}$ & $\begin{array}{l}\text { Diabetic } \\
n=7(4)\end{array}$ & $\begin{array}{l}\text { Control } \\
n=9(5)\end{array}$ & $\begin{array}{c}\text { Diabetic } \\
n=8(4)\end{array}$ & $\begin{array}{c}\text { Control } \\
n=10(5)\end{array}$ & $\begin{array}{c}\text { Diabetic } \\
n=10(5)\end{array}$ & $\begin{array}{c}\text { Control } \\
n=7(4)\end{array}$ & $\begin{array}{l}\text { Diabetic } \\
n=7(4)\end{array}$ & $\begin{array}{c}\text { Insulin } \\
n=8(4)\end{array}$ \\
\hline$T_{\max }\left(\mathrm{N} / \mathrm{cm}^{2}\right)$ & $25 \pm 2.2$ & $18 \pm 1.2$ & $25.5 \pm 2.5$ & $22.9 \pm 1.5^{\mathrm{b}}$ & $24.8 \pm 2.1$ & $20 \pm 2.2^{c}$ & $25.3 \pm 1.1$ & $15.2 \pm 1.2^{\mathrm{d}}$ & $24.7 \pm 2.3^{\mathrm{e}}$ \\
\hline Fatigue $\left(\% T_{\max }\right)$ & $16 \pm 2$ & $17 \pm 2$ & $10 \pm 1.5$ & $19.5 \pm 1^{\mathrm{b}}$ & $12 \pm 2$ & $24 \pm 2^{\mathrm{c}}$ & $15 \pm 1$ & $5 \pm 1^{\mathrm{d}}$ & $20 \pm 6^{\mathrm{e}}$ \\
\hline Recovery $\left(\% T_{\max }\right)$ & $80 \pm 5$ & $52 \pm 6^{a}$ & $62 \pm 4$ & $65 \pm 7$ & $70 \pm 2$ & $88 \pm 3^{\mathrm{c}}$ & $82 \pm 5$ & $39 \pm 4^{\mathrm{d}}$ & $78 \pm 6^{\mathrm{e}}$ \\
\hline \multicolumn{10}{|c|}{ Skinned fibers } \\
\hline & $n=12(3)$ & $n=9$ & & $n=8(3)$ & & $n=9(3)$ & & $n=12(4)$ & $n=12(4)$ \\
\hline$F_{\max }\left(\mathrm{N} / \mathrm{cm}^{2}\right)$ & $23 \pm 3.1$ & $17.3 \pm 2.2^{*}$ & & $21 \pm 1.1^{\#}$ & & $19.5 \pm 1.8^{\#}$ & & $14.5 \pm 2.8^{*}$ & $23.8 \pm 3.8^{\$}$ \\
\hline $\mathrm{pCa} 50$ & $5.62 \pm 0.03$ & $5.46 \pm 0.05^{*}$ & & $5.70 \pm 0.12^{\#}$ & & $5.87 \pm 0.02^{\#}$ & & $5.33 \pm 0.04^{*}$ & $5.63 \pm 0.07$ \\
\hline$N$ & $4.45 \pm 0.22$ & $3.55 \pm 0.09^{*}$ & & $3.25 \pm 0.22^{\#}$ & & $2.85 \pm 0.12^{\#}$ & & $3.35 \pm 0.20^{*}$ & $4.23 \pm 0.18$ \\
\hline
\end{tabular}

$n$ represents the number of individual muscle preparations studied while the number in parenthesis indicates the number of animals from which the fibers were isolated.

${ }^{\mathrm{a}} T_{\max }$ and recovery decreased in diabetic muscles $(P<.05)$

${ }^{\mathrm{b}} T_{\max }$ decreased and recovery enhanced in diabetic muscles $(P<.05)$

${ }^{\mathrm{c}} T_{\max }$ decreased, fatigue reduced and recovery enhanced in diabetic muscles $(P<.05)$

${ }^{\mathrm{d}} T_{\max }$ decreased, fatigue increased and recovery decreased in diabetic muscles $(P<.05)$

${ }^{\mathrm{e}} T_{\max }$, fatigue and recovery returned to control levels in diabetic muscles treated with insulin $(P<.05)$

${ }^{*} F_{\max }$ reduced, pCa50 reduced (less sensitive) and $N$ decreased (reduced cooperativity) in diabetic fibers $(P<.05)$

${ }^{\#} F_{\text {max }}$ reduced, pCa50 increased (more sensitive) and $N$ decreased (reduced cooperativity) in diabetic fibers $(P<.05)$

${ }^{\$} F_{\max }$, pCa50 and $N$ returned to control levels in diabetic fibers treated with insulin $(P<.05)$.

control muscles. Unlike the 4-day diabetic muscles strips that recovered to a lesser degree than the control muscle strips, after 4 weeks of diabetes, the muscles from these animals recovered to the same extend as the age-matched controls. Interestingly, by 4 weeks, diabetic muscles fatigued significantly less than the age-matched controls. The adaptation to lack of insulin persisted through 8 weeks of diabetes (Table 1, Figure 2(c)); the diabetic muscles fatigued even less and recovery from fatiguing stimulation became nearly complete (88\% of $T_{\max }$ versus $70 \%$ in the age-matched controls). $T_{\max }$ was further reduced in 8 -week diabetic muscles to approximately $20 \%$ of control levels. However, this seemingly beneficial adaptive response pattern to fatiguing stimulation dramatically changed at 14 weeks of diabetes (Table 1 , Figure 2(d)); all three contractile properties significantly decreased when compared to the age-matched controls. By 14 weeks, $T_{\max }$ was drastically reduced by $40 \%$ in diabetic muscles. We found that these late-stage responses to diabetes were completely reversed by insulin. When insulin was administered for two weeks to a set of animals diabetic for 12 weeks, $T_{\max }$ and force at the end of the fatiguing and recovery periods all returned to the levels observed in the age-matched controls.

While our intact muscle experiments were able to reveal clear temporally related phenotypic changes in DPH muscles from diabetic rats, it is difficult to infer from these types of experiments any cellular mechanisms that might account for the observed responses. Triton-skinned muscle fibers provide a suitable model system to identify specific modifications within the contractile machinery/proteins that are occurring at various times after streptozotocin treatment, particularly considering the temporal changes in $T_{\max }$ we detected in our studies. Figure 3 shows the force-versus-pCa relationships for single skinned muscle fibers from control (nondiabetic, 3 months of age), 4-day diabetic, 4-week, 8week diabetic, 14-week diabetic, and insulin-treated animals. At 4 days, the maximum calcium-activated force $\left(F_{\max }\right)$ significantly decreased (by 25\%) in a manner that closely matched the observed reduction in $T_{\max }$ of intact muscles (Table 1). In addition, the calcium concentration at which half-maximal activation was achieved $\left(\mathrm{Ca}_{50}\right)$ significantly increased, indicating that the fibers had become less sensitive to calcium (see Table 1, Figure 3). The slope of the curve $(N)$ also significantly decreased in the 4 -day diabetic fibers. Just as the intact diabetic muscle strips improved their performance after 4 and 8 weeks of diabetes, single skinned fibers from diabetic animals recovered to a significant extent from the loss in $F_{\max }$ (reduced by $\sim 9 \%$ at 4 weeks and $15 \%$ at 8 weeks) while their sensitivity to calcium progressively increased. It is interesting to note that a lower $F_{\max }$ and an increased calcium sensitivity are observed in slow-twitch, oxidative muscles as compared to fast-twitch, glycolytic muscles $[15,18]$. The slope of the force-versus-pCa curves continued to be reduced at these time points. As observed in intact muscles, this adaptive response was lost at 14 weeks after diabetes onset, with $F_{\max }$ and calcium sensitivity decreasing significantly. Muscles from 12-week diabetic animals treated for 2 weeks with insulin showed a dramatic change in the characteristics of their skinned fibers; the deterioration of function observed in 14-week diabetic animals was completely reversed with $F_{\max }, \mathrm{Ca}_{50}$, and $N$ values returning to control levels.

We sought to determine potential molecular mechanisms that might explain the adaptive responses of the muscles to diabetes. The reduction in force (both $T_{\max }$ and $F_{\max }$ ) and the increase in calcium sensitivity suggest a change in fiber type. Therefore, we performed Western blot analysis 


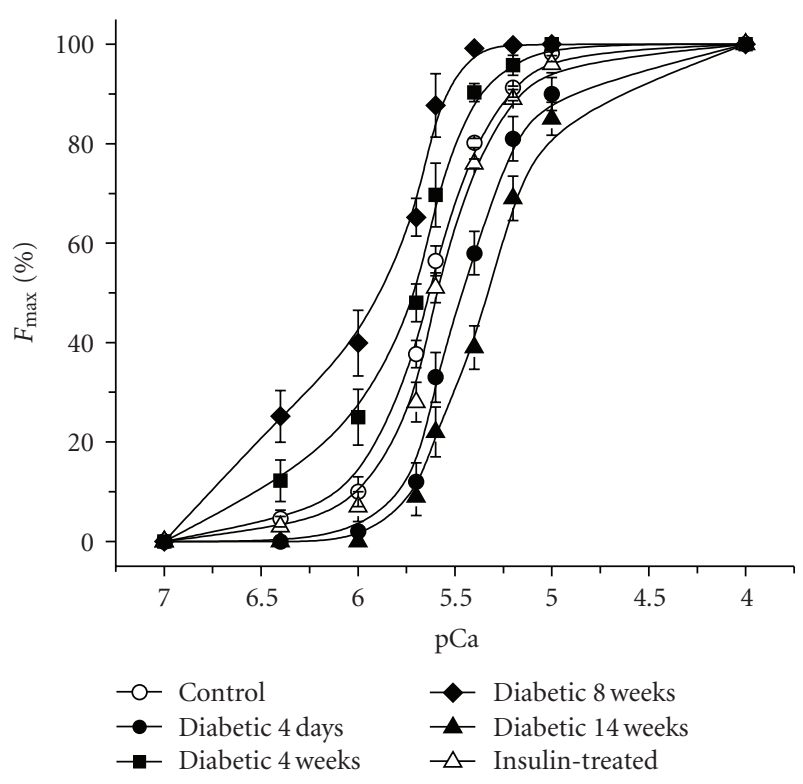

Figure 3: Diabetes alters the essential contractile properties in Triton-skinned muscle fibers. All data are presented as the mean \pm SD. Force-versus-pCa relationships for all conditions are shown. The open circle symbol shows the average control force-versus-pCa curve, closed circles show 3-4 days diabetic, closed squares 4 weeks diabetic, closed diamonds 8 weeks diabetic, closed triangles 14 weeks diabetic, and open triangles the insulin-treated muscle fibers. $F_{\max }$, number of experiments and statistical details are presented in Table 1 and methods.

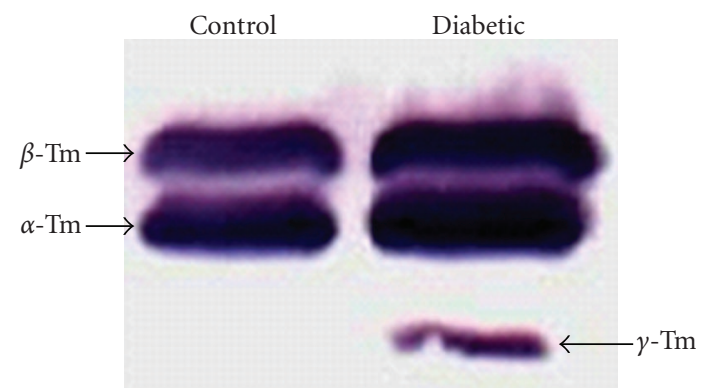

Figure 4: Expression of the slowest Tm isoform occurs in untreated DPH muscles after 8 weeks of diabetes. Western blot shows the control and diabetic expression patterns for all three Tm isoforms. The slowest Tm isoform is only detected in 8-week diabetic muscle homogenates. See experimental details in Section 2.

of tropomyosin $(\mathrm{Tm})$ isoforms in muscle homogenates from control and 8-week diabetic rats (Figure 4). We discovered that while control muscle samples only contained the $\alpha$ - and $\beta$-Tm isoforms, 8 -week diabetic samples contained the $\gamma$-Tm isoform, an isoform which uniquely appears in slow-twitch muscles [19].

\section{Discussion}

To our knowledge, this is the first study to investigate the effects of diabetes induced by STZ on DPH muscles from mammals during a sequential time frame extending from 4 days to 14 weeks. Our results clearly demonstrate that diabetes causes a detrimental acute effect on the ability of intact DPH muscles to generate maximal tetanic force ( $T_{\max }$ was decreased by $28 \%$ ), a condition where the contractile proteins are maximally stimulated with saturating intracellular calcium. Because the isolated muscle strips used in this study are directly stimulated, any effect of diabetes on force generation would be due to a decrease in the forcegenerating capability of the contractile apparatus and not to effects on either nerve activity or the neuromuscular junction. In fact, when single DPH fibers were isolated from the same muscles, maximum calcium-activated force $\left(F_{\max }\right)$ was reduced by essentially the same amount (25\%). By 4 weeks after streptozotocin treatment, both $T_{\max }$ and $F_{\max }$ significantly recovered but were still less than control values. It would appear that the contractile proteins are adapting to the diabetic state. However, by 14 weeks post streptozotocin, there was a major loss in the force-generating capabilities of the contractile proteins, both $T_{\max }$ and $F_{\max }$ decreased by approximately $40 \%$. Therefore, the myofilament protein adaptation is incapable of chronically compensating muscle function in diabetic conditions. This loss in force is due to altered glucose metabolism and/or lack of circulating insulin since DPH muscles from 12-week diabetic animals supplemented with insulin for 2 weeks presented a complete reversal of this detrimental effect. A recent study in musclespecific $G$ protein $\alpha$-subunit $\left(\mathrm{G}_{\mathrm{s}} \alpha\right)$ knockout mice [20] showed that this disruption of insulin signaling in skeletal muscles resulted in an adaptive fiber-type switch toward increased slow fiber contents in the absence of hyperglycemia. The results in our present study are consistent with this observation and further demonstrate a beneficial effect of the increase in slow fibers on compensating fatigue tolerance in diabetes.

Diabetic patients exhibit increased muscle fatigability and difficulty in squatting and in performing repetitive motions. Therefore, we stimulated the isolated DPH muscle strips from control and diabetic animals with a pattern of fatiguing stimulation to determine how they respond to, and recover from, the stress of increased activity. Both slow- and fast-twitch muscle fibers typically respond to fatiguing stimulation with a decrease in tetanic force that will recover to a varying degree upon cessation of stimulation [21]. Loss of force during the fatigue period can be due to (1) loss of the maximum force-producing capability of the contractile apparatus, (2) a decrease in the calcium sensitivity of the contractile apparatus, (3) disruption of the excitation-contraction coupling process that provides calcium to the contractile proteins, and (4) depletion of energy substrates, including ATP, creatine phosphate, and glucose. We found that after 4 days of diabetes, $T_{\max }$ decreases to the same extent after the fatiguing period when compared to age-matched control muscles. However, the fact that $T_{\max }$ does not recover to the same extent in diabetic as in control muscles signifies that one or more of these effects of fatiguing stimulation is/are irreversible in the diabetic muscles, at least at this time point. Irreversible damage of fatiguing stimulation to 
DPH muscle can account not only for possible respiratory complications but also for the increased muscle fatigue and propensity to inactivity experienced by many diabetic patients.

An adaptation to diabetes similar in time course to that observed for $T_{\max }$ and $F_{\max }$ was also observed in the response of the muscles to fatiguing stimulation. After 4 weeks of diabetes, the intact muscles fatigued less and recovered to the same extent as age-matched controls. After 8 weeks of diabetes, the fatigue response was significantly less than at 4 weeks of diabetes and the recovery was now significantly greater in the diabetic animals. The observation that the calcium sensitivity of the skinned fibers at both 4 and 8 weeks after diabetes was greater than control may at least partially account for this observation; even if the excitationcontraction coupling (ECC) process is permanently compromised by fatiguing stimulation, the increased calcium sensitivity of the fibers would limit the loss of tetanic force at the end of the recovery period. The potential beneficial effects of this adaptation are lost by 14 weeks post diabetes. At this time, corresponding to years of diabetes in humans, intact fibers fatigue to only $5 \%$ of the maximal $T_{\max }$ and the recovery is only to approximately $40 \%$. The corresponding loss of calcium sensitivity of the contractile proteins and the loss of cooperativity between the contractile proteins (as reflected in the decreased slope of the force-versus-calcium curve) can contribute to this response. Our functional data at the extreme periods studied here (4 days and 14 weeks) are in complete agreement with the findings of Hida et al. [12] and McGuire and MacDermott [13], respectively. Interestingly, all of these detrimental effects of diabetes on muscle function were completely reversed by 2 weeks of insulin treatment in our study, demonstrating the fundamental role of insulin signaling in maintaining skeletal muscle function. Whereas these benefits were concurrent with renormalized glycemia, which minimizes protein glycation and AGEs generation, the role of insulin-mediated cell signaling warrants further investigation.

Based on data available in the literature and on the progressive decrease in absolute force developed by DPH muscles from diabetic rats, it is conceivable that part of the process activated by diabetes involves alterations/adaptations in the contractile proteins. Contractile force is directly related to intracellular $\mathrm{Ca}^{2+}$ levels. The more calcium is released from the sarcoplasmic reticulum (SR), the higher the contractile force becomes due to the saturation of contractile proteins by $\mathrm{Ca}^{2+}$ (see Figure 3). It is evident from this relationship that the contractile force produced at higher frequencies of stimulation mirrors the events associated with the contractile proteins, while SR-related events are better reflected at the ascending part of the curve [22]. Because our studies were conducted under conditions where the frequency of stimulation used produced maximal tetanic force $\left(T_{\max }\right)$, we can postulate that the adaptation observed in DPH muscles from diabetic rats is probably intrinsic to the contractile proteins although other steps of the ECC process cannot be completely ruled out by our present study. This is supported by our studies on skinned fibers. The adaptive response during weeks $4-8$ of diabetes is similar to what one would find if the fibers with the diaphragm were switching from primarily fast-twitch to primarily slowtwitch, $F_{\max }$ would decrease and the fibers would become more sensitive to calcium [23]. We tested this hypothesis by measuring the isoforms of $\mathrm{Tm}$ that were present in control and diabetic muscles. $\alpha$ - and $\beta$-Tm isoforms are characteristic of fast-twitch muscles and we found only these isoforms in the control muscles. In contrast, 8-week diabetic muscle samples in addition to the $\alpha$ - and $\beta$ Tm isoforms contained the $\gamma$-Tm isoform, which is only found in slow-twitch muscle fibers [19]. While this is not a thorough analysis of isoforms changes in the contractile and regulatory proteins under these conditions, the studies of Zhi et al. [17] suggest that an increase in the $\gamma$-Tm isoform would be accompanied by an increase in the myosin heavy chain isoforms I and IIa, a decrease in the myosin heavy chain isoforms IIb and IIx, and a switch from fast to slow isoforms of Troponin I and Troponin T. Therefore, it would appear that at least part of the compensation observed at 4 and 8 weeks of diabetes is due to a certain degree of fiber-type switching. While this adaptation is potentially beneficial in that it can help the diaphragm by functional compensation, it is incapable of sustaining diaphragm function while diabetic lesion progresses, which will ultimately cause an uncompensated loss of function. The fiber-type switching, however, may represent only one aspect of the decreasing compensatory adaptation over time. Changes in energy metabolism, calcium cycling within the muscle fibers, diabetes progression, increased formation of reactive oxygen species, and AGEs products can all constitute causes or concauses of the observed phenomenon. It is very encouraging to note, however, that the loss of function can be fully reversed by insulin treatment. This finding is in agreement with the clinical observation that diabetic patients report fewer complaints about muscle fatigue when on insulin therapy and glucose levels are normalized [24].

\section{Conclusions}

In conclusion, our study is the first to indicate the occurrence of significant temporal adaptations in diaphragm contractility in type I diabetic animals. This change is triphasic, in that an initial deterioration is followed by a marked amelioration and by a tardy stage in which muscle function is again severely impaired. Our studies in skinned muscle fibers suggest that the cellular site of perturbation in diabetic muscle fibers is the contractile machinery/proteins. While our inaugural biochemical studies indicate the appearance of a slow tropomyosin isoform, additional biochemical approaches are required to more precisely determine the cellular basis of these changes in diabetic diaphragm as well as their pathophysiological significance. Protein modification might be an adaptive response to the diabetic milieu stress, while insulin therapy and glucose normalization might protect the contractile machinery against the deleterious effects of the oxidative and inflammatory milieu induced by diabetes. 


\section{References}

[1] V. Y. Polotsky, J. A. Wilson, A. S. Haines, et al., "The impact of insulin-dependent diabetes on ventilatory control in the mouse," American Journal of Respiratory and Critical Care Medicine, vol. 163, no. 3, part 1, pp. 624-632, 2001.

[2] M. A. Babcock, D. F. Pegelow, C. A. Harms, and J. A. Dempsey, "Effects of respiratory muscle unloading on exercise-induced diaphragm fatigue," Journal of Applied Physiology, vol. 93, no. 1, pp. 201-206, 2002.

[3] H. Andersen, "Motor function in diabetic neuropathy," Acta Neurologica Scandinavica, vol. 100, no. 4, pp. 211-220, 1999.

[4] H. W. Sander and S. Chokroverty, "Diabetic amyotrophy: current concepts," Seminars in Neurology, vol. 16, no. 2, pp. $173-178,1996$.

[5] R. R. Henry, "Glucose control and insulin resistance in non-insulin-dependent diabetes mellitus," Annals of Internal Medicine, vol. 124, no. 1, part 2, pp. 97-103, 1996.

[6] F. W. Booth and D. R. Vyas, "Genes, environment, and exercise," Advances in Experimental Medicine and Biology, vol. 502, pp. 13-20, 2001.

[7] N. Khandoudi, A. C. Guo, M. Chesnais, and D. Feuvray, "Skinned cardiac fibres of diabetic rats: contractile activation and effects of 2,3-butanedione monoxime (BDM) and caffeine," Cardiovascular Research, vol. 27, no. 3, pp. 447-452, 1993.

[8] I. Murat, V. L. Veksler, and R. Ventura-Clapier, "Effects of halothane on contractile properties of skinned fibers from cardiomyopathic animals," Journal of Molecular and Cellular Cardiology, vol. 21, no. 12, pp. 1293-1304, 1989.

[9] I. Murat and R. Ventura-Clapier, "Effects of halothane on skinned cardiac fibers from the myopathic hamster and the diabetic rat," Annales Francaises d'Anesthesie et de Reanimation, vol. 8, supplement, p. R137, 1989.

[10] G. M. M. Stephenson, A. O'Callaghan, and D. G. Stephenson, "Single-fiber study of contractile and biochemical properties of skeletal muscles in streptozotocin-induced diabetic rats," Diabetes, vol. 43, no. 5, pp. 622-628, 1994.

[11] V. I. Veksler, I. Murat, and R. Ventura-Clapier, "Creatine kinase and mechanical and mitochondrial functions in hereditary and diabetic cardiomyopathies," Canadian Journal of Physiology and Pharmacology, vol. 69, no. 6, pp. 852-858, 1991.

[12] W. Hida, C. Shindoh, J. O. Satoh, et al., "N-acetylcysteine inhibits loss of diaphragm function in streptozotocin-treated rats," American Journal of Respiratory and Critical Care Medicine, vol. 153, no. 6, pp. 1875-1879, 1996.

[13] M. McGuire and M. MacDermott, "The influence of streptozotocin-induced diabetes and the antihyperglycaemic agent metformin on the contractile characteristics and the membrane potential of the rat diaphragm," Experimental Physiology, vol. 83, no. 4, pp. 481-487, 1998.

[14] M. de Paula Brotto, S. A. van Leyen, L. S. Brotto, J.-P. Jin, C. M. Nosek, and T. M. Nosek, "Hypoxia/fatigue-induced degradation of troponin I and troponin C: new insights into physiologic muscle fatigue," Pflugers Archiv European Journal of Physiology, vol. 442, no. 5, pp. 738-744, 2001.

[15] M. A.P. Brutto, R. Y. Nagaraj, L. S. Brotto, H. Takeshima, J. Ma, and T. M. Nosek, "Defective maintenance of intracellular $\mathrm{Ca}^{2+}$ homeostasis is linked to increased muscle fatigability in the MG29 null mice," Cell Research, vol. 14, no. 5, pp. 373-378, 2004.

[16] M. A. P. Brotto and T. M. Nosek, "Hydrogen peroxide disrupts $\mathrm{Ca}^{2+}$ release from the sarcoplasmic reticulum of rat skeletal muscle fibers," Journal of Applied Physiology, vol. 81, no. 2, pp. 731-737, 1996.

[17] B. Y. Zhi, F. Gao, Z. F. Han, and J.-P. Jin, "Differential regulation of myofilament protein isoforms underlying the contractility changes in skeletal muscle unloading," American Journal of Physiology, vol. 292, no. 3, pp. C1192-C1203, 2007.

[18] N. Weisleder, M. Brotto, S. Komazaki, et al., "Muscle aging is associated with compromised $\mathrm{Ca}^{2+}$ spark signaling and segregated intracellular $\mathrm{Ca}^{2+}$ release," Journal of Cell Biology, vol. 174, no. 5, pp. 639-645, 2006.

[19] W. A. LaFramboise, R. C. Jayaraman, K. L. Bombach, et al., "Acute molecular response of mouse hindlimb muscles to chronic stimulation," American Journal of Physiology, vol. 297, no. 3, pp. C556-C570, 2009.

[20] M. Chen, H.-Z. Feng, D. Gupta, et al., " $\mathrm{G}_{\mathrm{s}} \alpha$ deficiency in skeletal muscle leads to reduced muscle mass, fiber-type switching, and glucose intolerance without insulin resistance or deficiency," American Journal of Physiology, vol. 296, no. 4, pp. C930-C940, 2009.

[21] M. A. P. Brotto, M. T. Marrelli, L. S. Brotto, M. Jacobs-Lorena, and T. M. Nosek, "Functional and biochemical modifications in skeletal muscles from malarial mice," Experimental Physiology, vol. 90, no. 3, pp. 417-425, 2005.

[22] M. A. de Paula Brotto, T. M. Nosek, and R. C. Kolbeck, "Influence of ageing on the fatigability of isolated mouse skeletal muscles from mature and aged mice," Experimental Physiology, vol. 87, no. 1, pp. 77-82, 2002.

[23] M. A. Brotto, B. J. Biesiadecki, L. S. Brotto, T. M. Nosek, and J.-P. Jin, "Coupled expression of troponin $\mathrm{T}$ and troponin I isoforms in single skeletal muscle fibers correlates with contractility," American Journal of Physiology, vol. 290, no. 2, pp. C567-C576, 2006.

[24] A. I. Vinik and Q. Zhang, "Adding insulin glargine versus rosiglitazone: health-related quality-of-life impact in type 2 diabetes," Diabetes Care, vol. 30, no. 4, pp. 795-800, 2007. 

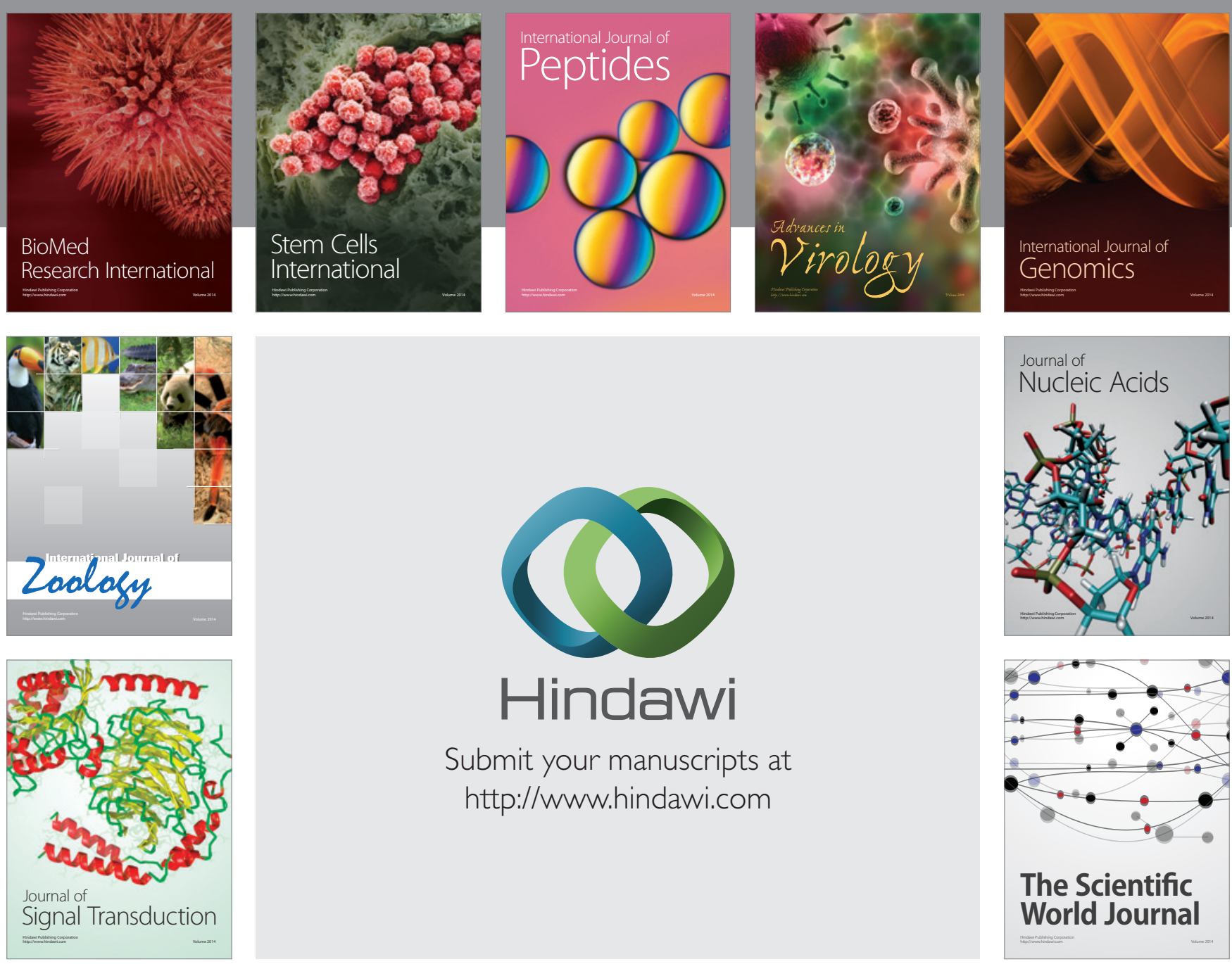

Submit your manuscripts at

http://www.hindawi.com
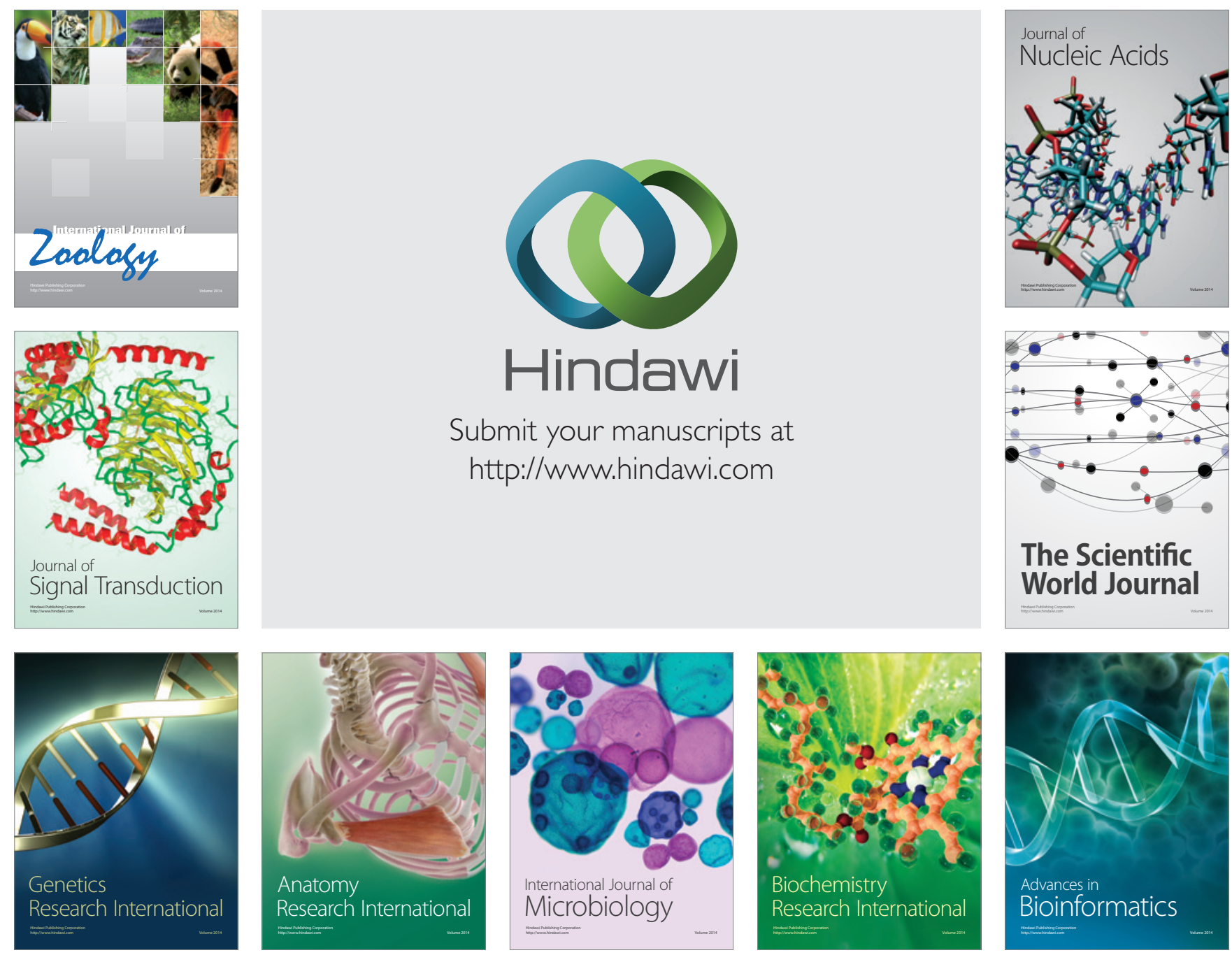

The Scientific World Journal
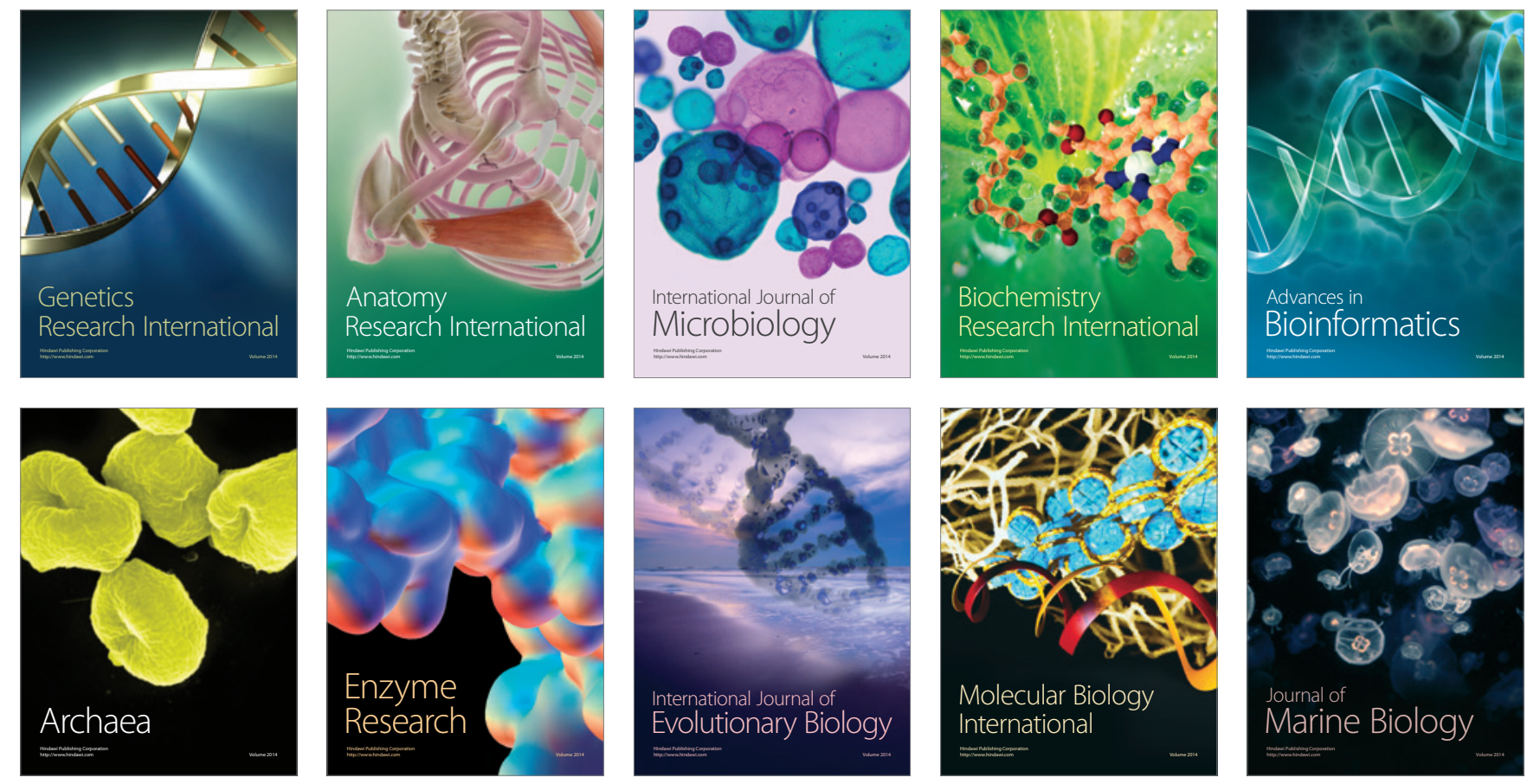